\section{Solvent Substitution Evaluation of Limestone Water as a Medium for Benzoylation}

\section{Abstract}

We herein reports limestone water as an alternate less basic medium for benzoylation of some aromatic compounds containing amino and hydroxyl functionality. Compared to traditional methodology, the technique we invented devoid of using organic or inorganic costlier hazardous alkaline solvents.

Keywords: Benzoylation; Limestone water; Industrial technique; Solvent substitution

\section{Deepak Chowrasia and Nisha Sharma}

\author{
University Institute of Pharmacy, CSJM \\ University, Kanpur-208024, India
}

\section{Corresponding author:}

Deepak Chowrasia

\section{chowrasia.deepak@gmail.com}

University Institute of Pharmacy, CSIM University, Kanpur-208024, India.

\section{Tel: +91-9451-019049}

Citation: Chowrasia D, Sharma N. Solvent Substitution Evaluation of Limestone Water as a Medium for Benzoylation. Arch Chem Res. 2016, 1:1.

\section{Materials and Methods}

All the reagents and solvents used in this experimentation are acquired from common store University Institute of Pharmacy, CSJM University, Kanpur, India and used as and their basis without any modification unless or until specified. The limestone used for preparation of water for benzoylation was purchased from Kidwai Nagar marble market, Kanpur, India and was used unmodified. Since compounds synthesised to evaluate limestone water for elucidating its feasibility for benzoylation are reported and spectrally characterized hence a comparative study between synthesized and reported compounds were done to enumerate its practicability and data regarding same is reported in Table 1 including supplementary file uploaded herewith. The melting point for synthesised compounds was recorded by open capillary method in triplicate and is uncorrected. The progression of reaction was monitored in PET ether:ethylacetate (8:2) as a binary solvent system. chemical transformation subsequently into amide ( $\mathrm{Ar}^{\prime} \mathrm{CONHAr} / \mathrm{R}$ ) or ester derivatives ( $\mathrm{Ar}^{\prime} \mathrm{CO}-\mathrm{OAr} / \mathrm{R}$ ) in the presence of alkaline solvents such as aqueous solution of sodium hydroxide or pyridine [2-6] along with benzoylating agent-benzoyl chloride or their substituted derivatives (Schotten-Baumann reaction) $[7,8]$. High melting point, resistance towards hydrolysis in aqueous medium, and insolubility of benzoylated derivatives makes the technique of benzoylation advantageously distinguished among acetylation (introduction of RCO-group), thus preferred comparatively $[7,8]$.

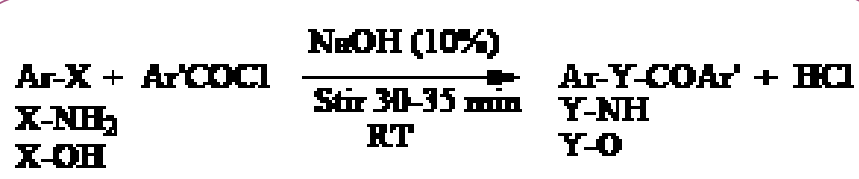

Scheme 1 Introduction of RCO-group. 


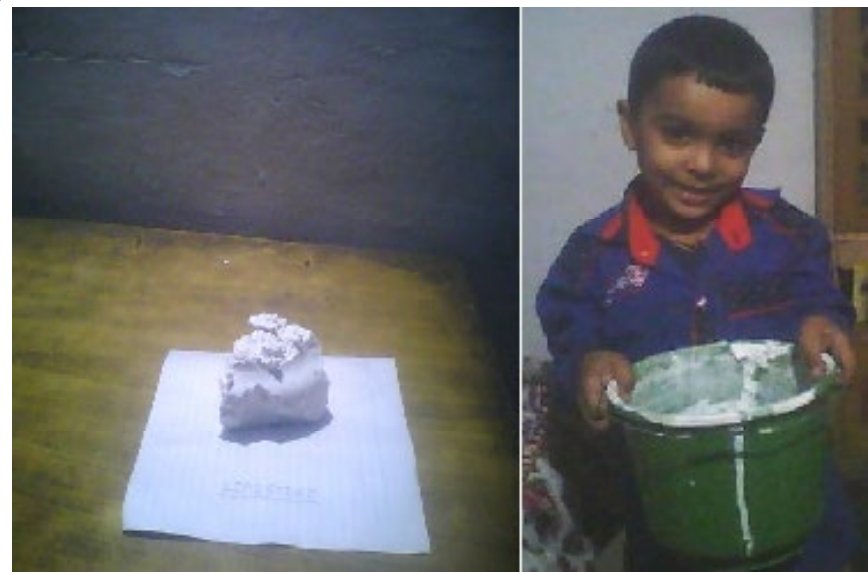

Figure 1 Limestone (left) and a child holding limestone water (Right).

\section{Experimental}

The efficiency of limestone water as a medium for synthesizing benzoylated derivatives was evaluated by dissolving or suspending equimolar quantity $(0.01 \mathrm{M})$ of reactants and benzoyl chloride in limestone water. The content was shaken for the sufficient period of time, yielding crude product, was further washed thoroughly from cold water and finally recrystallized from ethanol.

\section{Note}

Equimolar quantities of reactants and benzoyl chloride (slightly in excess amount and dropwise) was transferred in a well cleaned conical flask under the fuming hood. The flask was corked securely and resultant mixture vigorously shaken at room temperature until the product precipitate out from solution or odour of benzoyl chloride cease to evolve. In case if not precipitated, the flask was immersed in ice-chest or kept aside at room temperature until product is obtained. The crude product is filtered off and washed thoroughly with cold water followed by a biphasic mixture containing equal quantity of water and methanol. Once dried properly the same get recrystallized initially from water:methanol $(1: 1)$ and finally from ethanol.

\section{Results and Discussion}

The solvent substitute technique for benzoylation we herein develops and reported is unique and advantageous over conventional benzoylation methodology in terms of cost effectiveness, eco-compatibility, and is entirely free from using any harmful strong basic medium. The limestone water was successfully evaluated to elucidate its synthetic harmony for benzoylating monocarbocyclic as well as polycarbocyclic substituted and unsubstituted aromatic compounds containing hydroxyl and amino functionality. We further claims that the technique we herein reported can equally be applied for yielding benzoylated derivatives of aliphatic compounds containing hydroxyl and amino groups including protecting $\mathrm{N}$-terminal ends of amino acids for peptide synthesis however the same was reserved as a future workup plan of ours. Among synthesized compounds, $1 \mathrm{~b}$ was yielded in greater amount comparatively followed by $1 \mathrm{e}, 1 \mathrm{~d}, 1 \mathrm{~g}$, and $1 \mathrm{a}$ (Supplementary File). However compound if and 1c (Supplementary File) were obtained in smaller quantities. Furthermore, evaluating limestone water towards completion of reaction it was found that benzoylated derivatives obtained from mononuclear ring system was yielded sooner than those obtained from polynuclear ring system except $1 \mathrm{~g}$ and $1 \mathrm{f}$ (Supplementary File). The overall mean reaction time for mono and polycyclic ring system was calculated and found to be 12 and 23-minutes respectively. However when calculated individually the compound $1 \mathrm{~b}, 1 \mathrm{~d}$, and $1 \mathrm{e}$ (Supplementary File) were synthesized within a time interval of 10 -minutes; $1 \mathrm{~b}$ was the fasted yielded compound with an overall time duration of 4-minutes; else were synthesized within a time range of 13-22 minutes, $1 \mathrm{f}$ (Supplementary File) being the last to obtain ( 6 days).

To conclude the above phenomenon and get insight the differential reactivity pattern of these compounds structure oriented study was further included, was found that aromatic monocyclic ring system containing - $\mathrm{OH} /-\mathrm{NH}$ (Tables 2 and 3) functionality was easily benzoylated compared to their polycyclic analogues this may be because of distribution of electrons among single and polynuclear ring system. However when compared among, ring containing - NH functionality reacts rapidly with higher yield of final product (1b) than those containing $-\mathrm{OH}$ group. This may be assumed due to difference in reactivity pattern of functional group with benzoylating agent-benzoyl chloride (Tables 4 and 5).

\section{Conclusion}

Though overall yield of benzoylated derivatives we found in limestone water is not comparable with traditional alkaline solvents nevertheless its practicability in terms of cheap and ecocompatible solvent is undeniable. Furthermore the limestone water if used along with other alkaline solvent the net cost for industrial production for yielding benzoylated derivatives could efficiently be minimized. Since limestone water is comparatively less basic than conventional alkaline solvents hence this technique could be used for benzoylation of amino acid without causing their racemization. However as per our prediction, the limestone water could be used efficiently for synthesis of compounds $1 \mathrm{~b}$ and 1d (Supplementary File) and with substantial modification for compounds $1 \mathrm{a}, 1 \mathrm{c}$, and $1 \mathrm{~g}$ (Supplementary File). 
Table 1 Generalized description of synthesized compounds (*days, R-Reported, F-Found).

\begin{tabular}{|c|c|c|c|c|c|c|c|}
\hline Host reactants & Benzoylating agent & Solvent & Product & Reaction time (min.) & $\begin{array}{c}\text { Melting } \\
\text { R }\end{array}$ & $\begin{array}{c}\text { nt }(\stackrel{\circ}{ }) \\
F\end{array}$ & Yield (\%) \\
\hline Phenol & Benzoyl chloride & Limestone water & Phenyl benzoate (1a) & 13 & 70 & 72 & 52 \\
\hline Aniline & Benzoyl chloride & Limestone water & Benzanilide (1b) & 4 & 162 & 161 & 74 \\
\hline 1-naphthol & Benzoyl chloride & Limestone water & $\begin{array}{l}\text { Naphthalen-1-yl benzoate } \\
\text { (1c) }\end{array}$ & 27 & 56 & 57 & 43 \\
\hline 2-naphthol & Benzoyl chloride & Limestone water & $\begin{array}{c}\text { Naphthalen-2-yl benzoate } \\
\text { (1d) }\end{array}$ & 19 & 107 & 106 & 63 \\
\hline 4-hydroxy acetanilide & Benzoyl chloride & Limestone water & $\begin{array}{l}\text { 4-Acetamidophenyl } \\
\text { benzoate (1e) }\end{array}$ & 9 & $?$ & 201 & 70 \\
\hline Vanillin & Benzoyl chloride & Limestone water & $\begin{array}{l}\text { 4-Benzoyloxy-3-methoxy } \\
\text { benzaldehyde (1f) }\end{array}$ & $6^{*}$ & 77 & 75 & 38 \\
\hline Resorcinol & Benzoyl chloride & Limestone water & $\begin{array}{l}\text { 3-hydroxy phenyl benzoate } \\
\text { (1g) }\end{array}$ & 22 & 135 & 133 & 55 \\
\hline
\end{tabular}

Table 2 Qualitative test for presence of free $-\mathrm{OH}$ group in synthesized products.

\begin{tabular}{|c|c|c|c|}
\hline Compounds & Test & Result & Inferences \\
\hline Phenyl benzoate (1a) & Ferric chloride test & Negative & $\begin{array}{l}\text { No free } \mathrm{OH} \text { group present; } \\
\text { benzoylation done }\end{array}$ \\
\hline Benzanilide (1b) & Ferric chloride test & Not applicable & Not applicable \\
\hline Naphthalen-1-yl benzoate (1c) & Ferric chloride test & Negative & $\begin{array}{l}\text { No free } \mathrm{OH} \text { group present; } \\
\text { benzoylation done }\end{array}$ \\
\hline Naphthalen-2-yl benzoate (1d) & Ferric chloride test & Negative & $\begin{array}{l}\text { No free } \mathrm{OH} \text { group present; } \\
\text { benzoylation done }\end{array}$ \\
\hline 4-Acetamidophenyl benzoate (1e) & Ferric chloride test & Negative & $\begin{array}{l}\text { No free } \mathrm{OH} \text { group present; } \\
\text { benzoylation done }\end{array}$ \\
\hline $\begin{array}{l}\text { 4-Benzoyloxy-3-methoxy } \\
\text { benzaldehyde (1f) }\end{array}$ & Ferric chloride test & Negative & $\begin{array}{l}\text { No free } \mathrm{OH} \text { group present; } \\
\text { benzoylation done }\end{array}$ \\
\hline 3-hydroxy phenyl benzoate (1g) & Ferric chloride test & Violet color & $\begin{array}{l}\text { Single free } \mathrm{OH} \text { group present } \\
\text { benzoylation done }\end{array}$ \\
\hline Phenyl benzoate (1a) & Phthalein test & Negative & $\begin{array}{l}\text { No free } \mathrm{OH} \text { group present; } \\
\text { benzoylation done }\end{array}$ \\
\hline Benzanilide (1b) & Phthalein test & Not applicable & Not applicable \\
\hline Naphthalen-1-yl benzoate (1c) & Phthalein test & Negative & $\begin{array}{l}\text { No free } \mathrm{OH} \text { group present; } \\
\text { benzoylation done }\end{array}$ \\
\hline Naphthalen-2-yl benzoate (1d) & Phthalein test & Negative & $\begin{array}{l}\text { No free } \mathrm{OH} \text { group present; } \\
\text { benzoylation done }\end{array}$ \\
\hline 4-Acetamidophenyl benzoate (1e) & Phthalein test & Negative & $\begin{array}{l}\text { No free } \mathrm{OH} \text { group present; } \\
\text { benzoylation done }\end{array}$ \\
\hline $\begin{array}{l}\text { 4-Benzoyloxy-3-methoxy } \\
\text { benzaldehyde (1f) }\end{array}$ & Phthalein test & Negative & $\begin{array}{l}\text { No free } \mathrm{OH} \text { group present; } \\
\text { benzoylation done }\end{array}$ \\
\hline 3-hydroxy phenyl benzoate (1g) & Phthalein test & Light green color & $\begin{array}{l}\text { Single free } \mathrm{OH} \text { group present } \\
\text { benzoylation done }\end{array}$ \\
\hline
\end{tabular}


Table 3 Qualitative test for presence of free $-\mathrm{NH}$ group in synthesized products.

\begin{tabular}{|c|c|c|c|}
\hline Compounds & Test & Result & Inferences \\
\hline Phenyl benzoate (1a) & Dye test & Not applicable & Not applicable \\
\hline Benzanilide (1b) & Dye test & Negative & $\begin{array}{c}\text { No free }-\mathrm{NH}_{2} \text { group is present; } \\
\text { benzoylation done }\end{array}$ \\
\hline Naphthalen-1-yl benzoate (1c) & Dye test & Not applicable & Not applicable \\
\hline Naphthalen-2-yl benzoate (1d) & Dye test & Not applicable & Not applicable \\
\hline 4-Acetamidophenyl benzoate (1e) & Dye test & Not applicable & Not applicable \\
\hline $\begin{array}{l}\text { 4-Benzoyloxy-3-methoxy } \\
\text { benzaldehyde (1f) }\end{array}$ & Dye test & Not applicable & Not applicable \\
\hline 3-hydroxy phenyl benzoate (1g) & Dye test & Not applicable & Not applicable \\
\hline Phenyl benzoate (1a) & Bleaching sol test & Not applicable & Not applicable \\
\hline Benzanilide (1b) & Bleaching sol test & Negative & $\begin{array}{c}\text { No free }-\mathrm{NH}_{2} \text { group is present; } \\
\text { benzoylation done }\end{array}$ \\
\hline Naphthalen-1-yl benzoate (1c) & Bleaching sol test & Not applicable & Not applicable \\
\hline Naphthalen-2-yl benzoate (1d) & Bleaching sol test & Not applicable & Not applicable \\
\hline 4-Acetamidophenyl benzoate (1e) & Bleaching sol test & Not applicable & Not applicable \\
\hline $\begin{array}{l}\text { 4-Benzoyloxy-3-methoxy } \\
\text { benzaldehyde (1f) }\end{array}$ & Bleaching sol test & Not applicable & Not applicable \\
\hline 3-hydroxy phenyl benzoate (1g) & Bleaching sol test & Not applicable & Not applicable \\
\hline
\end{tabular}

Table 4 Solubility profile of synthesized compounds in different solvents.

\begin{tabular}{|c|c|c|c|c|c|c|c|c|}
\hline Compounds & Water & $\begin{array}{l}\mathrm{NaOH} \\
(10 \%)\end{array}$ & $\begin{array}{c}\mathrm{NaHCO}_{3} \\
(10 \%)\end{array}$ & Acetic acid(10\%) & $\begin{array}{c}\mathrm{HCl} \\
(10 \%)\end{array}$ & $\begin{array}{c}\text { Methanol } \\
(50 \%)\end{array}$ & $\begin{array}{l}\text { Ethanol } \\
(100 \%)\end{array}$ & $\begin{array}{c}\text { Chloroform } \\
\text { (100\%) }\end{array}$ \\
\hline Phenyl benzoate (1a) & - & + & + & -+ & + & + & +++ & +++ \\
\hline Benzanilide (1b) & - & - & - & -+ & + & ++ & +++ & +++ \\
\hline $\begin{array}{l}\text { Naphthalen-1-yl benzoate } \\
\text { (1c) }\end{array}$ & - & - & - & -+ & -+ & + & +++ & +++ \\
\hline $\begin{array}{l}\text { Naphthalen-2-yl benzoate } \\
\text { (1d) }\end{array}$ & - & - & - & -+ & -+ & + & +++ & +++ \\
\hline $\begin{array}{l}\text { 4-Acetamidophenyl } \\
\text { benzoate (1e) }\end{array}$ & -+ & -+ & - & -+ & -+ & ++ & +++ & +++ \\
\hline $\begin{array}{l}\text { 4-Benzoyloxy-3-methoxy } \\
\text { benzaldehyde (1f) }\end{array}$ & - & - & - & -+ & -+ & ++ & +++ & +++ \\
\hline $\begin{array}{l}\text { 3-hydroxy phenyl benzoate } \\
\text { (1g) }\end{array}$ & -+ & -+ & - & -+ & -+ & ++ & +++ & +++ \\
\hline
\end{tabular}


Table 5 Structure of synthesized compounds.

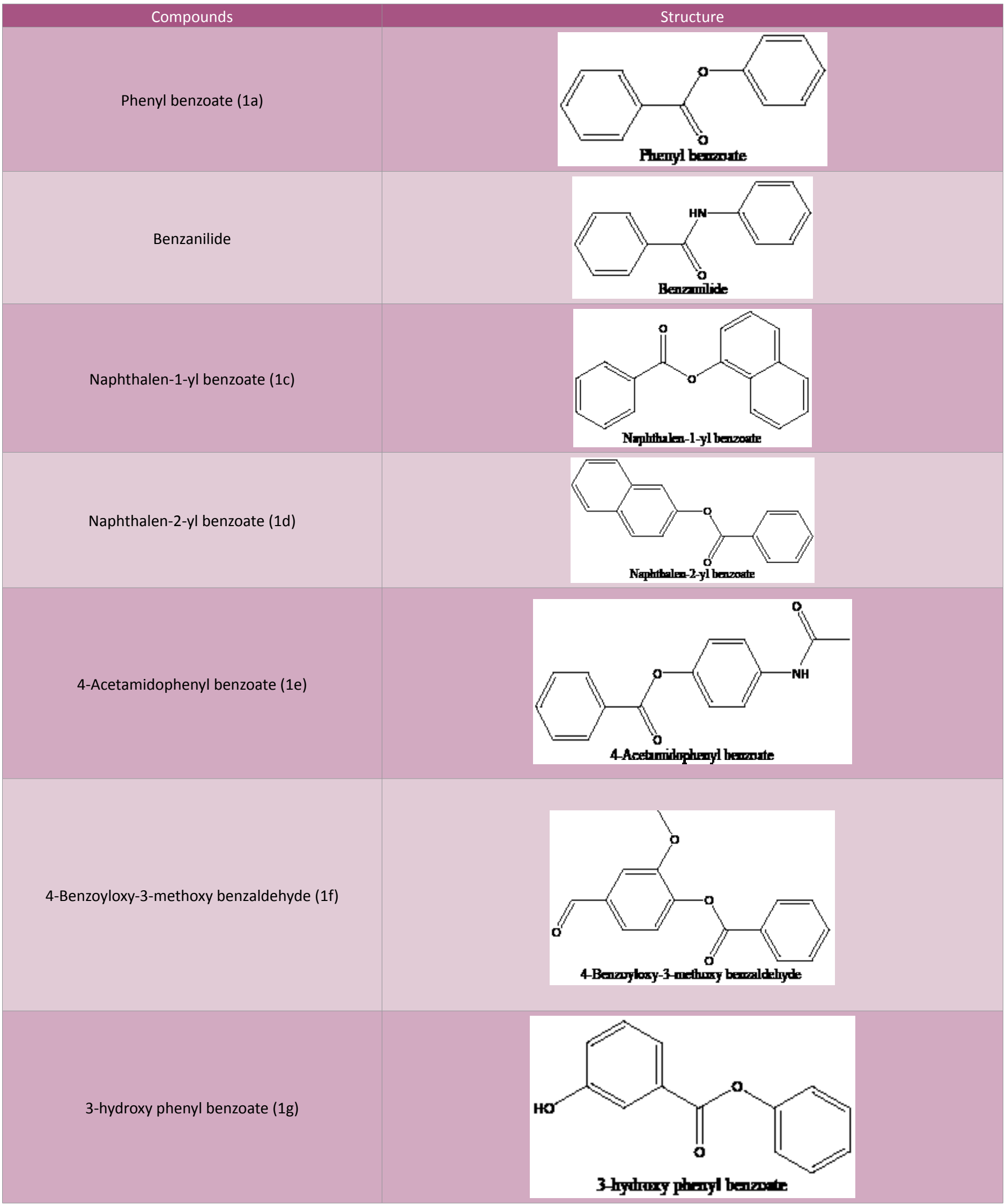




\section{References}

1 Tapia O, Bertran J (2002) Solvent effect and chemical reactivity. Kluwer academic publisher, Dordrecht p: 17.

2 Greene TW (1981) Protective groups in organic synthesis, Wiley NY, USA pp: 261-263.

3 Reese CB (1973) Protective groups in organic chemistry, Plenum press London, UK pp: 52-53.

4 Ando W, Tsumaki H (1983) A facile preparation of aliphatic hydroxamic acid from $\mathrm{N}, \mathrm{N}, \mathrm{O}$-tris(trimethylsilyl)hydroxylamine and acid chloride. Synthetic communications 13: 1053-1056.
5 Taylor EC, Mclay GW, McKillop A (1968) Thallium in organic synthesis. II. Acylation, Aroylation, and tosylation, of phenol and carboxylic acid. Journal of American chemical Society 90: 2422-2423.

6 Illi VO (1979) Phase transfer catalyzed acylation. Tetrahedron Letters 20: 2431-2432.

7 Vogel Al (1956) Practical organic chemistry: Longman group limited London pp: 582-583.

8 Mann FG, Saunders BS (1978) Practical organic chemistry, Longman group limited London pp: 243-244. 